


\title{
EL BIOCLUB COMO ESTRATEGIA PARA LA CONSERVACIÓN DE LA TORTUGA MORROCOY, GEOCHELONE CARBONARIA (SPIX 1824) EN CURUMANÍ $(\text { CESAR })^{1}$
}

\section{The Bioclub as a Strategy for the Conservation of the Morrocoy Tortoise, Geochelone carbonaria (Spix 1824) In Curumaní-Cesar.}

\author{
Mary Lorena Moyano Acevedo ${ }^{2}$ \\ Jeisson Steven López Oliva ${ }^{3}$
}

Fecha de recepción: 03 de marzo de 2014

Fecha de aprobación: 19 de mayo de 2015

\section{Resumen}

En esta investigación, se presentan las implicaciones pedagógicas que se generan a partir de la implementación de la estrategia educativa nombrada "Bioclub", con respecto a las prácticas que tiene la población del municipio de Curumaní en el departamento del Cesar (Colombia), con respecto a la tortuga morrocoy. A través de la recolección de información referente a las condiciones socioeconómicas de la comunidad se diseñó la estrategia educativa, siguiendo la premisa del aprendizaje contextualizado. En una segunda etapa se aplicaron las sesiones del Bioclub, donde directamente participó la comunidad, representada por estudiantes del Colegio San José, para la obtención de datos relacionados con las prácticas frente a la especie, así como su reconocimiento biológico y ecológico. Por último, se caracterizan dichas prácticas de la comunidad en relación con la tortuga, las implicaciones referentes al desarrollo de una estrategia educativa enfatizando en los beneficios que tiene el club de ciencias en el proceso enseñanza/aprendizaje, en la construcción del conocimiento, y cómo esto puede generar un cambio positivo en las actitudes de los niños en el cuidado ambiental y la conservación de la biodiversidad colombiana. Por último se desarrolló una Cartilla de conservación construida como resultado del Bioclub, que involucra aspectos relacionados con su biología y ecología, además de una guía encaminada a la cría sostenible, con el fin de minimizar el impacto negativo de las prácticas de la comunidad frente a la especie.

\footnotetext{
1 Trabajo de grado para optar al título de Licenciados en Biología. Universidad Pedagógica Nacional.

2 Licenciada en Biología de la Universidad Pedagógica Nacional.

3 Licenciado en Biología de la Universidad Pedagógica Nacional.
} 


\section{Abstract}

In this research, the pedagogical implications generated from the implementation of educational strategy named Bioclub with respect to practices that have the population of the municipality of Curumaní in the department of Cesar (Colombia) are presented regarding the Tortuga Morrocoy. Through the collection of information on the socioeconomic conditions of the community education strategy was designed on the premise of contextualized learning. In a second step Bioclub meetings where the community participated directly represented by students of San José College, to obtain data related practices concerning species and their biological and ecological survey were applied. Lastly, the practices of the community in relation to the turtle, the implications concerning the development of an educational strategy emphasizing the benefits that science club in the teaching-learning process in the construction of knowledge are characterized, and how this may generate a positive change in the attitudes of children in environmental stewardship and conservation of biodiversity in Colombia. Finally we developed a Conservation Primer built as a result of Bioclub involving aspects of their biology and ecology, as well as a guide aimed at sustainable farming, in order to try to minimize the negative impact of the practices of the community to species.

\section{Introducción}

A partir de las diferentes experiencias que se presentan al cursar la carrera de Licenciatura en Biología, en la Universidad Pedagógica Nacional, fue constante la presencia de dos problemáticas a las que se enfrenta en la actualidad el profesor de biología. La primera de ellas tiene que ver con la creciente pérdida de biodiversidad del mundo y en especial la del territorio colombiano por los diferentes problemas ambientales que afectan los ecosistemas, los cuales se ven perturbados por las prácticas humanas frente al consumo desenfrenado de los recursos para satisfacer necesidades egoístas. La segunda problemática está relacionada con la situación del sistema educativo tradicional, evidenciado en los niveles de deserción y analfabetismo que siguen siendo altos en el país, y aunque en cuanto a datos y porcentajes se muestra una supuesta disminución, no hay ningún índice o estadística que demuestre el aparente desinterés de los niños y niñas por asistir al colegio a aprender.

Debido a lo anterior, desde la línea de investigación "Concepciones acerca de la vida y de lo vivo en contextos culturalmente diferenciados", el trabajo de grado "El Bioclub como estrategia para la Conservación de la tortuga morrocoy, Geochelone carbonaria (spix 1824) en Curumaní, Cesar", busca brindar una alternativa que genere un cam- bio o una posible solución frente a estas situaciones, por medio de una estrategia educativa distinta enmarcada en el aprendizaje no formal, como lo es un club de ciencias, qué para el desarrollo de esta investigación tiene como objetivo fomentar el cuidado hacia todo lo vivo, razón por la cual fue llamado "Bioclub".

Dentro del Bioclub se contextualizaron los procesos educativos como aspecto primordial para la construcción del conocimiento, por lo que fue necesario trabajar en pro de la conservación de una especie casi endémica del país y con gran arraigo en la cultura de la población, que posiblemente se está viendo afectada en gran medida por las prácticas humanas. Por esta razón, se escoge a la tortuga morrocoy (Geochelone carbonaria) que en la actualidad se encuentra en estado crítico de conservación según la Unión Internacional para la Conservación de la Naturaleza (IUCN, por su sigla en inglés), y además se establece el desarrollo del Bioclub en el municipio de Curumaní en el departamento del Cesar, ubicado en la costa Atlántica colombiana. Allí, esta especie se relaciona con las costumbres y prácticas de la comunidad; con el consumo de su carne y huevos; con la elaboración de artesanías e instrumentos musicales con su caparazón, y con la comercialización ilegal de la especie, entre otros elementos.

En este documento se presentan las implicaciones pedagógicas que se generan a partir de la implementación de la estrategia educativa Bioclub, con respecto a las prácticas que tiene la población del municipio de Curumaní, en el departamento del Cesar, frente a la tortuga morrocoy. Se hace énfasis en los beneficios de la estrategia del club de ciencias en el proceso enseñanza/aprendizaje, frente a la construcción del conocimiento, y cómo esto puede generar un cambio positivo en las actitudes de los niños, es decir las generaciones futuras, frente al cuidado ambiental y la conservación de la biodiversidad.

Para ello, en primera instancia, se recolectó información referente a las condiciones socioeconómicas de la comunidad, necesarias para el diseño de la estrategia educativa, a partir de la premisa del aprendizaje contextualizado; a esta primera parte se le denominó fase de contextualización.

En la segunda etapa se aplicaron las sesiones del Bioclub, en las que participó la comunidad de forma directa, representada por estudiantes del Colegio San José. Aquí se obtuvieron datos acerca de las prácticas que tienen frente a esta tortuga, así como algunas características biológicas y ecológicas que fueron observadas por los niños.

Al finalizar se caracterizaron las prácticas que posiblemente generan algunas de las problemáticas que afectan 
las poblaciones de la especie en la localidad, además de todas las implicaciones referentes al desarrollo de una estrategia educativa diferente tanto en las actitudes de los estudiantes como en la búsqueda de soluciones frente a las problemáticas anteriormente mencionadas. Así pues, se obtuvieron las herramientas necesarias para el planteamiento de una propuesta de conservación sobre la especie, que involucró aspectos relacionados con su biología y ecología, además de una guía encaminada a la cría sostenible, con el fin de minimizar el impacto negativo de las prácticas de la comunidad frente a la especie.

La investigación se desarrolló en dos etapas: la primera de ellas referente al trabajo en campo y la segunda al análisis de la información obtenida. Durante la primera etapa se realizó la fase de contextualización del municipio y de la institución educativa por medio de la encuesta social y la entrevista no estructurada, con el fin de diseñar las sesiones del Bioclub. Posteriormente, se llevó a cabo la fundamentación -donde se trabajó con los estudiantes diferentes temáticas en torno a aspectos biológicos y ecológicos de la especie-, y la problematización -donde se hizo un acercamiento a las problemáticas que afectan a G. carbonaria a partir de la perspectiva de los niños-.

Como principales resultados se evidenció que sí es posible generar una mayor apropiación de los saberes y una construcción visible del conocimiento, a través de una alternativa de enseñanza diferente a la escuela tradicional, mediante actividades que estén diseñadas bajo la premisa del aprendizaje contextualizado, debido al mayor impacto que se genera en los niños. Además es evidente un interés significativo frente a la estrategia y las actividades que allí se realizan, demostrado en la activa y constante participación, el sentido de pertenecían adquirido por el espacio y por la posibilidad de expresar sentimientos, opiniones y argumentos que ayuden a alcanzar un objetivo común; sin dejar de lado, la elaboración de una posible solución, como lo es la propuesta de conservación, que está contextualizada para su municipio y enmarcada en sus propias necesidades, haciendo que la educación tenga una finalidad inmediata y que los conocimientos que se construyen sean de utilidad real.

\section{Materiales y métodos}

Para responder a los objetivos mencionados, se enfocó el trabajo en un paradigma interpretativo, cuya finalidad, como la enuncia González (2000), es comprender e interpretar acciones humanas, prácticas sociales y teorías interpretativas. Estas últimas tratan del estudio de la sociedad visto imparcialmente en donde el investigador se debe convertir en uno solo con la comunidad estudiada para así entenderla mejor. Por lo mismo, la finalidad de este paradigma es hacer un acercamiento a la comprensión e interpretación de una realidad, así como de los significados, percepciones, intenciones y acciones de las personas de una comunidad.

Además se hizo uso de una herramienta, cuya implementación y sistematización, ayudó en la obtención e interpretación de los datos. Esta herramienta fue la encuesta social, por medio de la cual se recolectó información con respecto al estrato social, algunas características correspondientes a la familia, como, número de integrantes, tipo de vivienda, ubicación; y otras relacionadas con el nivel de educación, profesión, entre otras.

\section{Métodos de campo: investigación cualitativa}

Para el desarrollo de esta investigación se consideraron algunos métodos como la observación participante (Galeano, 2007), que es una estrategia en la cual los investigadores tienen una relación directa con la comunidad o la población del estudio, en este caso con las prácticas. Para ello se buscó un acercamiento con las diferentes prácticas que presenta la comunidad de Curumaní frente a la tortuga morrocoy (G. carbonaria), este método ayudó a encontrar y analizar las diferentes costumbres y comportamientos de la comunidad con respecto a la especie, mediante charlas y observaciones de las rutinas de los pobladores, así como durante el desarrollo de las sesiones del Bioclub, en la etapa de la fase de contextualización.

La entrevista (López y Deslauriers, 2011), la cual es una estrategia donde el investigador, por medio de una discusión estructurada o no estructurada con otra persona, obtiene información más precisa sobre el objeto de estudio. Para este trabajo de investigación, las entrevistas fueron utilizadas durante la fase de contextualización y problematización, al igual que la encuesta social. La encuesta social (Sarduy, 2007), fue una herramienta utilizada por los investigadores para obtener información directamente de la población, usada durante la fase de contextualización, tanto al inicio de las sesiones del Bioclub, como al finalizar las mismas, la cual permitió obtener fundamentos que para el caso de un enfoque cualitativo (Galeano, 2007), sirvieron durante el análisis de los datos, que fueron obtenidos con otras estrategias como la observación participante, la entrevista y los grupos de discusión.

Por último, los grupos de discusión, como lo enuncia Briones (1996), son una estrategia de investigación que plantea la formación o la integración de un grupo de personas que discuten acerca de un tema específico, en donde el investigador cumple el papel de facilitador y mediador, lo que permite que la discusión sea libre con 
argumentos espontáneos, con un discurso natural y no impuesto. Para este trabajo de investigación, los grupos de discusión fueron utilizados durante el desarrollo del Bioclub, en algunas sesiones, donde se trabajó con los estudiantes participantes, con respecto a las prácticas de la comunidad con la especie G. carbonaria, incitando a la discusión y análisis sobre las mismas.

\section{Métodos de sistematización}

El proceso de registro y sistematización de la información recolectada en una investigación cualitativa es el paso intermedio entre la obtención y generación de los datos, para su posterior análisis. El registro riguroso de estos datos ayudó a poner en orden el conjunto de información generado en el proceso de investigación, para que su análisis fuera rápido y eficaz.

La sistematización rigurosa, con técnicas y herramientas pertinentes (teniendo en cuenta los métodos descritos por Galeano, 2001) como el desarrollo de tablas, matrices y gráficos, permitieron un rápido manejo de la información, así como la revisión de los datos que para la investigación, se consideraron más importantes y apropiados. En este trabajo de investigación, con el fin de sistematizar la información obtenida en el proceso investigativo, direccionar su análisis y facilitar su revisión y posterior socialización, se establecieron una serie de modelos y estrategias de registro y sistematización de información, las cuales estuvieron directamente relacionadas con un tipo de investigación cualitativa, como es el caso del registro en el diario de campo, las fotografías y los videos.

El registro de la información recolectada en el trabajo de campo (entrevistas, observación participativa, grupos de discusión) fue consignado de manera descriptiva, en lenguaje natural (como lo hablaron las personas y los niños pertenecientes al Bioclub), para así contextualizar la información. Por ejemplo, en los debates y los grupos de discusión fue importante el uso de una videograbadora, la cual facilitó la sistematización de los diferentes discursos.

\section{Métodos de análisis}

El análisis de la información obtenida durante el proceso investigativo forma parte de la recuperación, adquisición y apropiación de todos aquellos conocimientos presentes en las distintas fuentes consultadas, y que tienen como objetivo principal identificar aquellos datos verdaderamente útiles y de mayor importancia para la finalidad del trabajo investigativo, por tal razón, para este trabajo de investigación se llevaron a cabo estrategias de análisis como la sistematización, la descripción, la categorización, la interpretación y el análisis de los datos a través del software NVIVO9.

En este proyecto de investigación se llevó a cabo la descripción de los resultados de cada sesión, por medio del análisis de los trabajos realizados, entre ellos cuentos, maquetas, collages e historietas construidas por los niños integrantes del Bioclub, a través de la interpretación de las historias y los dibujos que muestran las representaciones que tienen de la realidad construidas a partir de las prácticas sociales.

En la tabla 1 se muestran algunas categorías, determinadas a partir de los valores de uso de Primack (2010) y su porcentaje de tendencia en la primera encuesta. 


\begin{tabular}{|c|c|c|}
\hline Categoría & Respuestas & Porcentaje \\
\hline Valor de no uso. Valor de existencia. & $\begin{array}{l}\text { "Parte importante del medio". } \\
\text { "Es un ser viviente". } \\
\text { "Porque es parte de él y por lo cual debe ser importante". } \\
\text { "Porque son parte del medio ambiente". } \\
\text { "Porque son muy pequeñas y no dañan". } \\
\text { "Son animales que no hacen nada malo". } \\
\text { "Porque la tortuga tiene derecho a la libertad". } \\
\text { "Porque es un ser vivo". } \\
\text { "Porque es muy buena". } \\
\text { "Porque es parte de nuestro planeta y como tal debemos protegerla". } \\
\text { "Es un animal muy importante y es de vital importancia que no se } \\
\text { extinga". } \\
\text { "Es un animal". }\end{array}$ & $40 \%$ \\
\hline Valor de uso directo: valor consuntivo & $\begin{array}{l}\text { "Es una Fuente de alimento para nosotros". } \\
\text { "Pero solo para alimento". } \\
\text { "Porque son ricas". } \\
\text { "Porque ellos son una fuente de alimento para muchos". }\end{array}$ & $13,3 \%$ \\
\hline Valor de opción & $\begin{array}{l}\text { ¿Pueden ser útiles para nuestro futuro". } \\
\text { "Porque será bueno en un futuro". } \\
\text { "Porque son animales importantes para la vida del ser humano en } \\
\text { el mañana". } \\
\text { "Pueden ser útiles en un futuro". }\end{array}$ & $13,3 \%$ \\
\hline $\begin{array}{l}\text { Valor de uso indirecto: valor de uso no con- } \\
\text { suntivo }\end{array}$ & $\begin{array}{l}\text { "Son animales que ayudan a conservar el ecosistema". } \\
\text { "Sirven para el medio ambiente". } \\
\text { "Es un animal clave para el consumo de plagas". } \\
\text { "Porque ayudan a conservar el ecosistema". } \\
\text { "No ensucian el ecosistema". } \\
\text { "Porque es buena para el ecosistema". } \\
\text { "Es importante en la cadena alimenticia". }\end{array}$ & $23,3 \%$ \\
\hline $\begin{array}{l}\text { Valor de uso no consuntivo: Recreación y } \\
\text { ecoturismo. }\end{array}$ & $\begin{array}{l}\text { ¿Nos brinda alegría y compañía (mascota)". } \\
\text { ¿Son animales que muchas veces les trae alegría a los niños". } \\
\text { "Porque la tortuga es un animal de crianza". }\end{array}$ & $10 \%$ \\
\hline
\end{tabular}

Tabla 1. Categorización de los valores de conservación para G. carbonaria dados por la comunidad. Pregunta 7, Encuesta N. ${ }^{\circ}$. Bioclub "Salvadores de tortugas"; Curumaní (Cesar, Colombia). 
Así mismo, en el cuadro 1 se presentan las problemáticas que afectan la tortuga, enunciadas por los mismos estudiantes luego de varias sesiones del Bioclub.

\begin{tabular}{|c|c|c|}
\hline Problemática & Descripción & Análisis \\
\hline Consumo de carne y huevos. & $\begin{array}{l}\text { "No estamos seguros de qué edad tienen las tortu- } \\
\text { gas cuando las comemos". } \\
\text { "Aquí se come mucho la tortuga en semana santa } \\
\text { y en diciembre". } \\
\text { "Conozco gente que también come tortuga en } \\
\text { cumpleaños y bautizos". }\end{array}$ & $\begin{array}{l}\text { Consumo de la carne de la tortuga y sus hue- } \\
\text { vos en épocas del año específicas; consumo } \\
\text { indiscriminado de los ejemplares sin tener en } \\
\text { cuenta las edades de reproducción de la espe- } \\
\text { cie. }\end{array}$ \\
\hline Uso como mascota & $\begin{array}{l}\text { "Muchas personas ahogan a veces a la tortuguitas } \\
\text { por que las ponen en agua pensando que son de } \\
\text { agua". } \\
\text { "Las tortuguitas se mueren de hambre porque las } \\
\text { personas no saben qué darles de comer". } \\
\text { "Los pelaos pequeños no las dejan quietas cuando } \\
\text { son pequeñas [las tortugas] y las matan entecadas } \\
\text { o las espichan porque no se acuerdan dónde las } \\
\text { dejan". }\end{array}$ & $\begin{array}{l}\text { Tortugas que son adoptadas como mascotas } \\
\text { y viven bajo condiciones poco adecuadas que } \\
\text { puedan ocasionar escenarios considerados } \\
\text { por la especie inapropiados para la reproduc- } \\
\text { ción o el desovo. }\end{array}$ \\
\hline Incendios provocados & $\begin{array}{l}\text { "Un tío mío incendió el solar para desmontarlo } \\
\text { [quitar maleza] y vimos cuando muchos animales } \\
\text { salieron corriendo, al otro día encontramos varias } \\
\text { tortugas quemadas". }\end{array}$ & $\begin{array}{l}\text { Muchos organismos, incluyendo tortugas } \\
\text { pertenecientes a la especie, son sacrificados } \\
\text { durante las quemas provocadas para la defo- } \\
\text { restación intencional, que se lleva a cabo en } \\
\text { beneficio del pastoreo y la ganadería en la } \\
\text { región. También este proceso se da con el fin } \\
\text { de asustar los organismos, sacarlos de sus } \\
\text { madrigueras o escondites y facilitar su cap- } \\
\text { tura. }\end{array}$ \\
\hline Contaminación del hábitat & $\begin{array}{l}\text { "Al botar basura, estamos matando a muchos ani- } \\
\text { malitos, porque contaminamos los lugares donde } \\
\text { viven". }\end{array}$ & $\begin{array}{l}\text { La contaminación del ecosistema donde } \\
\text { habita la tortuga afecta el proceso de repro- } \\
\text { ducción y desovo. Además, muchos materia- } \\
\text { les pueden matar el organismo asfixiándolo o } \\
\text { ahorcándolo como las bolsas y otros produc- } \\
\text { tos de plástico. Incluso, sustancias utilizadas } \\
\text { en el proceso de curtiembre en el sector afec- } \\
\text { tan la calidad de los cuerpos de agua de los } \\
\text { que las tortugas se abastecen. }\end{array}$ \\
\hline $\begin{array}{l}\text { Transporte ilegal de la espe- } \\
\text { cie }\end{array}$ & $\begin{array}{l}\text { "A muchos animalitos como las tortugas se las lle- } \\
\text { van para Europa o Estados Unidos como mascotas, } \\
\text { pero son lugares donde hace mucho frío o no hay } \\
\text { lo que comen entonces se mueren, otras se mue- } \\
\text { ren en el viaje". }\end{array}$ & $\begin{array}{l}\text { En muchos casos, especies endémicas de } \\
\text { Suramérica son llevadas a otros continentes } \\
\text { como mascotas exóticas, privándolas de las } \\
\text { condiciones adecuadas para su superviven- } \\
\text { cia. }\end{array}$ \\
\hline
\end{tabular}

Cuadro 1. Caracterización de las problemáticas que afectan la tortuga morrocoy; sesión 17 Bioclub "Salvadores de tortugas"; Curumaní (Cesar, Colombia). 


\section{Fases metodológicas}

Con el fin de alcanzar los objetivos planteados en esta investigación, también se desarrollaron dos etapas generales: la primera de ellas referente al trabajo en campo y la segunda al análisis de la información obtenida, cabe aclarar que en muchas ocasiones el trabajo de las etapas fue simultáneo.

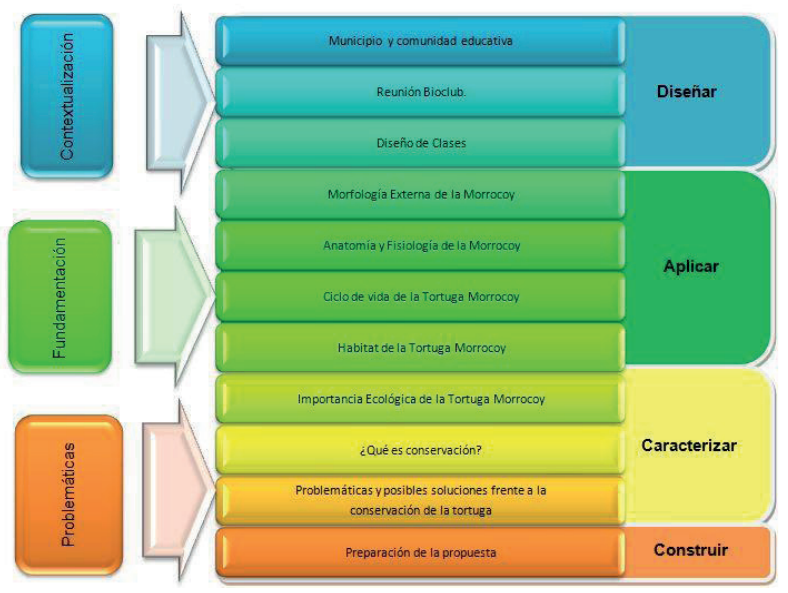

Figura 1. Esquema metodológico del proyecto de investigación "El Bioclub como estrategia para la conservación de la tortuga morrocoy, Geochelone carbonaria (Spix 1824) en Curumaní-Cesar".

Con el fin de caracterizar las prácticas de la comunidad se recopiló la información obtenida en algunas de las sesiones de la fase de fundamentación y toda la fase de problematización, además de la implementación de otra encuesta social y la entrevista no estructurada. En consecuencia, y como producto final del Bioclub, se construyó una cartilla educativa dirigida al público en general pero dirigida a los niños de la comunidad, como una propuesta de conservación para la tortuga morrocoy.

\section{Resultados y discusión}

El Bioclub se desarrolló en el Colegio San José, una institución educativa oficial ubicada en el casco urbano del municipio, con unas instalaciones amplias que albergan a cerca de novecientos estudiantes entre primaria y bachillerato. Al iniciar la convocatoria para la conformación del Bioclub se solicitó a la rectora del colegio establecer los cursos con los cuales se podía trabajar en el proyecto, para lo cual se asignó a los grados de quinto a séptimo. Los estudiantes fueron escogidos en un principio por la docente de Ciencias Naturales a cargo de dichos cursos, teniendo en cuenta algunos criterios en los estudiantes como el nivel de responsabilidad, participación, compromiso y disciplina.
Teniendo en cuenta los anteriores criterios, a la primera sesión, en la cual se explicó la naturaleza y objetivos del Bioclub, asistieron 15 niños; sin embargo para las siguientes el número definitivo de estudiantes fue de 23 , incluyendo uno de cuarto de primaria; no todos recomendados por la docente de Ciencias Naturales. Lo anterior relacionado con las dinámicas manejadas durante el Bioclub, por las temáticas trabajadas y las actividades desarrolladas, lo que pudo generar en los niños un interés considerable que impulsó su participación a lo largo del desarrollo de la propuesta. Por último, las edades de los niños oscilaron entre los 8 y los 15 años de edad; 14 mujeres y 9 hombres. La duración del Bioclub fue de 22 sesiones, un total de tres meses, con dos sesiones por semana: una sesión los martes y otra los jueves, con una duración de hora y media, es decir tres horas por semana.

En cuanto a la realización del Bioclub se evidenció que este funciona como una estrategia que fomenta el interés de los niños frente a la conservación de la biodiversidad y las especies como G. carbonaria, generando una fuerte relación entre el sentimiento de biofilia y los valores de la conservación.

Según Cubides y Romero (2012)

"Los clubes de ciencias nacen de la necesidad de que sean los mismos niños y jóvenes quienes indaguen sobre las preguntas que se plantean del mundo. Como nuevos espacios en la construcción del futuro; en estos grupos se desarrolla un proceso de aprendizaje del conocimiento científico, a partir de sus propios intereses"
El Bioclub plantea un esquema diferente al de la educa- ción formal, donde se adoptan características llamati- vas para los niños y niñas que por medio de actividades y dinámicas distintas se involucra el constante uso de la creatividad y el continuo desarrollo psicomotriz, lo que crea un aumento de interés y una construcción del conocimiento visible, diferente al que se da durante los procesos comunes en la escuela tradicional.

Frente a este aspecto, la inclusión de características propias de la didáctica de este tipo de estrategias como la autonomía, la autorregulación y el aprendizaje autodirigido influyen en la construcción de conocimiento, debido a que el estudiante "asume prioritariamente la responsabilidad para planificar (tiempo-espacio-ritmo), desarrollar y evaluar su aprendizaje" (Bolívar, 2004), hecho que marca de manera drástica los conocimientos que construya y como estos le sean útiles para usar en el entorno en el que vive. 
De lo anterior, también vale la pena rescatar la importancia de la contextualización de las dinámicas que se lleven a cabo durante el proceso enseñanza/aprendizaje, otra característica sobresaliente en el desarrollo del Bioclub, teniendo en cuenta que en el contexto, según Pratt (1997), "se ayuda a determinar la significatividad del acontecimiento con vista a su utilidad futura". Lo anterior fue evidente ya que la misma estructuración pedagógica de las sesiones del Bioclub permitió que los niños pudieran mostrar a lo largo del desarrollo de las actividades características propias de su cotidianidad, su entorno y su diario vivir, incluyendo así representaciones de sus prácticas, de sus personalidades, de sus jergas y dialectos, no solo frente a la especie, la biodiversidad del sector, sino también a sus relaciones familiares, educativas e interpersonales.

Por otro lado la implementación constante de actividades manuales, las cuales tienen gran acogida por los estudiantes, por encima de las escritas, lo cual está relacionado con el desarrollo psicomotriz que implica la elaboración de las manualidades, además de la posibilidad de expresar los sentimientos, emociones y propiciar el desarrollo de la creatividad; los trabajos escritos comunes implican la realización de una misma actividad en repetidas ocasiones, por el contrario las actividades manuales repercuten en el mantenimiento de las conexiones cerebrales, generando unas nuevas cada vez que se cambia la rutina de trabajo y la construcción del conocimiento se hace más evidente.

Otro de los beneficios observados al culminar el Bioclub como estrategia educativa es la expresión máxima del sentimiento de biofilia en los estudiantes integrantes, entendida como la "afiliación emocional innata que tienen los seres humanos a otros organismos vivos" (Wilson, 1993), lo anterior respecto a la afinidad de ellos frente a la protección de la especie $G$. carbonaria, evidente aún más en su interés por la construcción de la estrategia en pro de su conservación, que está relacionado con los valores de la conservación de no recursos definidos por Primarck (2010), como por ejemplo la apreciación del valor de existencia, luego de examinar las problemáticas que afectan en gran medida a la tortuga morrocoy.

Con respecto a lo anterior se contrastaron los resultados de las encuestas con la caracterización de las problemáticas expuestas por los niños al culminar las sesiones del Bioclub; esto demostró que las problemáticas presentadas por la comunidad en general son reconocidas por los niños, ya que se muestra una relación con la evidente caza de especies silvestres con fines de consumo de su carne, generalmente de juveniles, lo que ocasiona una posible interferencia en el ciclo de vida de la especie. Esta práctica está ligada a un comercio ilegal que, a su vez, se relaciona con un transporte ilícito que trasciende de la región o incluso del país. Además, los niños hicieron explícita una relación entre el deterioro del ambiente de la localidad y el detrimento de la especie, enfatizando en la culpabilidad de las labores humanas frente a este aspecto, que incluyen quemas de basura, incendios forestales provocados y contaminación de las quebradas.

Asimismo, a partir del trabajo realizado en el Bioclub, y siguiendo la tendencia que muestra que los niños prefieren las actividades manuales y lúdicas sobre las escritas, se propuso la cartilla Salvemos la tortuga morrocoy como una propuesta de conservación para la especie G. carbonaria, la cual incluye varias actividades planteadas y realizadas por los niños del Bioclub y por medio de las cuales se demostró que en los niños se generó un mayor interés frente al cuidado y conservación de la especie, una mayor apropiación de su entorno e inclusión de sí mismos en un ecosistema que es habitado por muchos otros organismos. Lo anterior es muestra de que el trabajo en el club de ciencias, siguiendo estrategias diferentes de enseñanza, aumenta el sentido de pertenecía frente a la naturaleza, el ambiente, los ecosistemas y la biodiversidad en general, apoyado también en la afirmación de Bazo (2011) de que en el club de Ciencias "se busca realizar actividades de educación y divulgación, con el propósito de despertar o incrementar el interés por la ciencia y la tecnología", y como lo dice la ACAC (2012) "Los Clubes de Ciencias constituyen una propuesta pedagógica estratégica para la formación y el desarrollo de los niños, niñas y jóvenes ya que fomentan aptitudes en la investigación y desarrollan habilidades científicas mediante el trabajo en equipo", así pues es fácil fomentar en los estudiantes que participen en el Bioclub actitudes referentes al cuidado del ambiente y sobre todo la conservación de la biodiversidad de la tierra, en la siguiente tabla se muestran los resultados relacionados con lo anterior, luego del desarrollo de una actividad en las últimas sesiones y que no era evidente al inicio del Bioclub. 


\begin{tabular}{|c|c|c|c|}
\hline $\begin{array}{l}\text { TIPO DE } \\
\text { OBRA }\end{array}$ & PERSONAJES & DESCRIPCIÓN & $\begin{array}{l}\text { CARACTERÍSTICAS } \\
\text { SOBRESALIENTES }\end{array}$ \\
\hline Títeres & $\begin{array}{l}\text { Tortuga Morrocoy, Águila, } \\
\text { niño e iguana. }\end{array}$ & $\begin{array}{l}\text { Se cuenta la historia de una tortuga que } \\
\text { lucha por poner sus huevos, pero que } \\
\text { es atacada por varios depredadores, sin } \\
\text { embargo, es protegida por un niño de } \\
\text { una iguana y un águila, por lo cual logra } \\
\text { poner a salvo los huevos de la tortuga. }\end{array}$ & $\begin{array}{l}\text { El humano juega un papel de protector para } \\
\text { la especie, aunque la depredación sigue } \\
\text { teniendo una perspectiva negativa. Se evi- } \\
\text { dencia que la tortuga hace parte de un eco- } \\
\text { sistema y dinámicas, dentro de la red trófica } \\
\text { de la zona. }\end{array}$ \\
\hline Títeres & $\begin{array}{l}\text { Tortuga Morrocoy, señora y } \\
\text { cazador. }\end{array}$ & $\begin{array}{l}\text { Se narra la historia de un cazador que } \\
\text { iba a tomar una tortuga de su medio } \\
\text { silvestre, la cual es protegida por una } \\
\text { señora que pasaba por el lugar, y recri- } \\
\text { mina las acciones de esta persona bajo } \\
\text { el argumento del valor estético de la } \\
\text { especie. Al finalizar la historia el cazador } \\
\text { agrede a la mujer y consigue llevarse a la } \\
\text { tortuga. "no se puede cazar las tortugas, } \\
\text { porque son muy lindas para nosotros los } \\
\text { seres humanos..." }\end{array}$ & $\begin{array}{l}\text { Se habla del valor estético que tiene la espe- } \\
\text { cie para los humano } \\
\text { La obra representa una de situaciones que se } \\
\text { diagnosticaron durante la contextualización } \\
\text { referente a la caza ilegal de la especie para } \\
\text { diversos fines. }\end{array}$ \\
\hline Títeres & $\begin{array}{l}\text { Liebre, zorro y Tortuga } \\
\text { Morrocoy. }\end{array}$ & $\begin{array}{l}\text { Es una obra que representa la fabula tra- } \\
\text { dicional de la liebre y la tortuga. }\end{array}$ & $\begin{array}{l}\text { Se resalta el papel que juega la tortuga den- } \\
\text { tro de un ecosistema y su interacción con } \\
\text { otros organismos, además de sus hábitos } \\
\text { de comportamiento, aunque cabe decir que } \\
\text { la moraleja de la fabula es no ser soberbio ni } \\
\text { confiado. }\end{array}$ \\
\hline Obra de teatro. & $\begin{array}{l}\text { Tortuga, guardabosques y } \\
\text { niña. }\end{array}$ & $\begin{array}{l}\text { La historia trata de una niña que arroja } \\
\text { basura en una zona donde viven tortu- } \\
\text { gas, y un guardabosque se da cuenta } \\
\text { antes de que la tortuga se vea afectada } \\
\text { por la basura. Durante la conversación } \\
\text { entre la niña y el guardabosque este le } \\
\text { informa que no debe arrojar basuras al } \\
\text { ambiente ya que la tortuga podría con- } \\
\text { sumir alguno objeto que pueda matarla, } \\
\text { a lo que la niña responde que "no me } \\
\text { interesa". }\end{array}$ & $\begin{array}{l}\text { Se resalta la gran influencia que tiene el } \\
\text { humano frente a los ecosistemas y las otras } \\
\text { especies, incluida la tortuga Morrocoy, ya sea } \\
\text { una influencia negativa, como en el caso de la } \\
\text { niña, o positiva como en el caso del guarda- } \\
\text { bosque. De esta manera se simula una situa- } \\
\text { ción muy común. } \\
\text { En la obra se hace mención al valor intrínseco } \\
\text { y valor de opción de G. carbonaria. }\end{array}$ \\
\hline
\end{tabular}

Cuadro 2. Categorización de las dramatizaciones: importancia ecológica; sesión 12 Bioclub "Salvadores de tortugas", Curumaní (Cesar, Colombia). 
Frente al quehacer docente, en el trabajo del Bioclub como una estrategia distinta, que es parte de la educación no formal, cabe afirmar que no es pertinente que el maestro adopte nuevos papeles distintos al de su propia naturaleza, intentado ser tomado como un guía o simple instructor, sino que se debe ver

[...] como un actor principal dentro del escenario educativo, el cual se asume como autónomo, creador, investigador e intelectual. De allí, que se conciba como aquel que se compromete con su labor, apropiándola desde su ser y su quehacer y orientándola hacia la formación de sujetos capaces de reflexionar y de tomar decisiones dentro de la comunidad a la que pertenecen. (Cubides y Romero, 2012,)

Por esta razón se debe asumir responsabilidad conjunta con los estudiantes en el diseño, desarrollo y evaluación de las actividades, para que si no se direcciona de manera radical el proceso, sí se coordine y se apoye a las decisiones de los estudiantes, además de brindarles de manera continua y apropiada los conocimientos para que sean alcanzados los objetivos planteados. Así, la construcción del conocimiento siempre será bidireccional, y el maestro estará en continuo aprendizaje.

Aunque la implementación de esta estrategia para fomentar el sentido de conservación en las nuevas generaciones puede llegar a ser difícil, relacionado con el condicionamiento a la escuela y a los procesos tradicionales de enseñanza-aprendizaje a los que los niños están acostumbrados, vale la pena intentar cambiar los métodos y las estrategias de enseñanza para incentivar en ellos nuevas formas de vida que no sólo los beneficien y a las personas que los rodean, sino a todas las especies y ecosistemas con quienes comparten la tierra.

\section{Conclusiones}

Se puede concluir que el Bioclub es una alternativa viable como estrategia educativa por que les permite a los niños ser partícipes activos del proceso enseñanza/aprendizaje, no solo en la aplicación de este, sino también en el diseño y evaluación de dicho proceso. Además se puede decir que el trabajo en el Bioclub aumenta en los niños el sentimiento de biofilia hacia la diversidad, siendo clave en la comprensión del sentido de la conservación de las especies y los ecosistemas.

Por otro lado las dinámicas desarrolladas en el club de ciencias permiten identificar de primera mano problemáticas relacionadas con el diario vivir de las personas y como en este caso con las prácticas de la comunidad de Curumaní (Cesar) con respecto a la especie G. carbo- naria, lo que facilita su caracterización y en gran medida la proposición de soluciones contextualizadas a dichas problemáticas, como por ejemplo la construcción de una cartilla como estrategia de conservación para la Tortuga Morrocoy elaborada a partir de los vivido en el Bioclub.
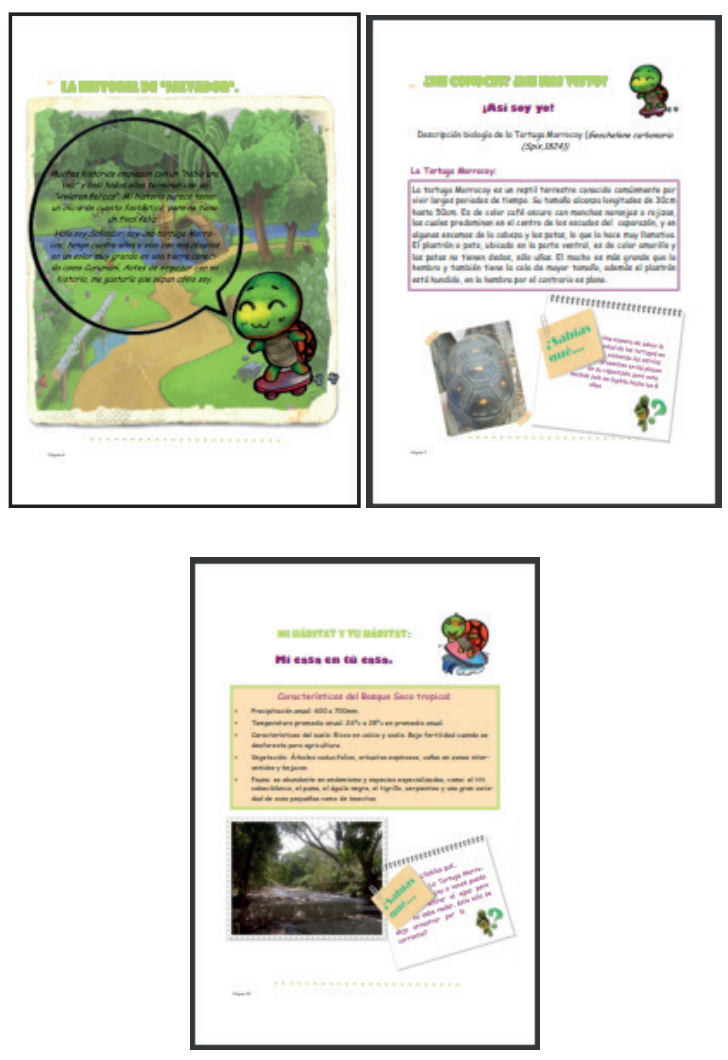

Figura 2. Páginas de la cartilla Salvemos la tortuga morrocoy. Estrategia de conservación diseñada teniendo en cuenta las sesiones del Bioclub y las propuestas de sus integrantes.

En cuanto a las actividades trabajadas a lo largo del Bioclub, se pudieron identificar los beneficios que presenta el trabajo manual con respecto al desarrollo de la creatividad, la imaginación, los valores personales y familiares, la personalidad y el pensamiento crítico. Así, se logra plasmar en los productos obtenidos características particulares de su cotidianidad, su contexto y su forma de vida, lo que facilita para el investigador la comprensión de las prácticas, las costumbres y los quehaceres. Por otro lado, para el niño este tipo de estrategia educativa es más llamativa por que logra extraerlo del contexto escolar tradicional y lo involucra en otro tipo de educación en la cual las actividades generan una apropiación de los saberes y una adecuada construcción del conocimiento.

Con respecto a las prácticas culturales que posee la comunidad frente a la tortuga Morrocoy se pudo establecer que existen cinco tendencias marcadas que afectan de manera clara el bienestar de la especie en la localidad. En primer 
lugar el consumo de la carne del animal, que para ser más claros no es en sí lo que genera un efecto negativo sino la falta de conocimiento frente al desarrollo de la misma práctica, debido a que se consumen huevos y jóvenes que no han llegado a el acto reproductivo, lo que fragmenta el ciclo de vida de la especie, generando a su vez la disminución de los ejemplares de la especie. En segunda instancia, la caza indiscriminada e ilegal, también relacionada con el transporte ilícito, apoyan en gran medida la disminución de ejemplares ya que son extraídos de su hábitat natural, incluyendo además el mantenimiento de la especie en cautiverio bajo condiciones inapropiadas que dificultan el progreso normal del ciclo vital y a su vez la expectativa de reproducción generando una baja en la tasa de natalidad y una reducción en la esperanza de vida de los organismos bajo estas condiciones. A lo anterior se le suma la contaminación y deterioro del hábitat de la tortuga Morrocoy, que dificulta en gran medida el desarrollo normal de los ejemplares en estado silvestre de la especie G. carbonaria.

Como una posible solución a las problemáticas que afectan de manera directa la especie, y que son generadas en gran medida por las practicas de la población, al finalizar

\section{Referencias bibliográficas}

Bazo, R. H. (2011). El club de ciencias y la indagación escolar. Ministerio de Ciencia, Tecnología e Innovación productiva. Edit. Mentes Inquietas Programa de Ciencia Tecnología y Educación. Buenos Aires, Argentina.

Bolívar, A. (2004). Diccionario Enciclopédico de la Didáctica. Archidona, Málaga: Ediciones Aljibe.

Briones, G. (1996). Metodología de la investigación. La encuesta social. Módulos de investigación social. Bogotá: Uniminuto, Icfes.

Cubides, E y Romero, Y. (2010). El club de ciencias: ¿por qué construirlo como una estrategia pedagógica? Revista Biografía 3(5). Pág. 125-154

Galeano Marín, M.E. (2001). Registro y sistematización de información cualitativa, Grupo Investigación Calidad de Vida. Interacciones y pensamientos. Explotación sexual infantil y juvenil: construcción de comunidad académica y avances investigativos. Medellín.

Galeano M., M.E. (2007). Estrategias de investigación social cualitativa: El giro en la mirada. Medellín: La Carreta Editores.

González, J. (2000). El paradigma interpretativo en la investigación social y educativa: Nuevas respuestas para viejos interrogantes. Sevilla: Universidad de Sevilla. el Bioclub se propone recurrir a la educación no formal a través de una cartilla educativa para fomentar en los lectores un sentimiento de conservación con el fin de disminuir los efectos negativos brindando herramientas para reconocer las tortugas morrocoy como organismos que hacen parte del entorno de la comunidad, su importancia ecológica y como pueden ser aprovechadas como "recurso" sin afectar todas las poblaciones silvestres y la especie en general. Así pues se propone una apropiación del conocimiento referente a la cría sostenible de la especie en los hogares de la comunidad y teniendo en cuenta las condiciones favorables para el organismo.

Por último, este tipo de trabajo investigativo ayuda a los maestros en formación, como nosotros, en el desarrollo de su que hacer frente a nuevas estrategias de enseñanza que incentiven la construcción del conocimiento que atienda a problemáticas contextualizadas frente a las necesidades sociales y ambientales. Además, la relación con las comunidades en las que se desarrolla la investigación presenta nuevas formas de ver el mundo y como vivirlo, aspectos que cambian o influyen directamente en la forma de enseñar.

López E., R.E. y Deslauriers, J.P. (2011). La entrevista cualitativa como técnica para la investigación en trabajo social. Margen, 61. Nuevo León, México.

Pratt, H. (1997) Diccionario de Sociología. 2a. ed. México: Fondo de Cultura Económica.

Primack, R.B. (2010). Essentials of Conservation Biology. 5a. ed. Sunderland, Massachusetts: Sinauer Associates, Inc.

QSR Internacional (2010). NVivo9. Microsoft Partner, Gold Independent Software Vendor (ISV). Estados Unidos.

Romero C., C. (2005). La categorización, un aspecto crucial en la investigación Cualitativa. Revista de Investigación Cesmag (11), 113-118.

Sarduy, Y. (2007), El análisis de información y las investigaciones cuantitativa y cualitativa. Revista Cubana de salud pública. Vol. 33 №3. Cuidad de la Habana, Cuba.

Wilson, E.O. (1993). Biophilia and the Conservation Ethic. En: S. Kellert S. y E.O. Wilson (eds.). The Biophilia Hypothesis. Shearwater Books (pp. 31-40). Editorial Island Press Washington. D.C. 